\title{
A method for designing lightweight and flexible creep-age forming tools using mechanical splines and sparse controlling points
}

\author{
Aaron C. L. Lam • Zhusheng Shi • Jianguo Lin • \\ Xia Huang • Yuansong Zeng • Trevor A. Dean
}

Received: 3 September 2014 / Accepted: 2 March 2015 /Published online: 24 March 2015

(C) The Author(s) 2015. This article is published with open access at Springerlink.com

\begin{abstract}
Creep-age forming (CAF) is a proven forming technique in the aerospace industry for the production of large integrally stiffened panels. One of the most urgent issues to be addressed in CAF is the development of flexible tooling. Flexible tools already have a long-standing reputation for the economic impact they have brought to the aircraft industry. However, with the rising need to establish comprehensive springback prediction models for CAF, the need for flexible CAF tools is now stronger than ever. In this article, an existing state-of-the-art CAF tool is described followed by the introduction of a novel design concept for flexible tooling. Based on the proposed design method, which utilises mechanical splines and sparsely spaced controlling points, a proof-of-concept prototype is built and characterised using corresponding analytical and finite element models that have been developed. Three parameters that can influence forming surface error: (i) the number of control points, (ii) spaces between control points and (iii) spline thickness are identified and optimised. Finally, an integrated optimisation process for tool offsetting is introduced, and its use is demonstrated. It is confirmed that this design method can be used to make flexible CAF tools with less than $\pm 1 \mathrm{~mm}$ error (defined as vertical difference from prediction) in the forming surface. In addition, this error can
\end{abstract}

A. C. L. Lam $\cdot$ Z. Shi $\cdot$ J. Lin $(\bowtie)$

Department of Mechanical Engineering, Imperial College London, London SW7 2AZ, UK

e-mail: Jianguo.Lin@Imperial.ac.uk

X. Huang $\cdot$ Y. Zeng

AVIC Beijing Aeronautical Manufacturing Technology Research

Institute, Beijing 100024, People's Republic of China

T. A. Dean

School of Mechanical Engineering, University of Birmingham,

Birmingham B15 2TT, UK eventually be compensated and thus eliminated from CA-formed parts by using the developed optimisation technique. This article provides CAF tool designers confirmed advices for making new flexible CAF tools. Lightweight and flexible CAF tools can now be constructed through the use of mechanical splines and sparse controlling points.

Keywords Creep-age forming (CAF) $\cdot$ Lightweight flexible CAF tools · Modular tool design · Tool offsetting algorithm . Inversed Macaulay's method · Euler-Bernoulli beam theory

\section{Nomenclature}

$a_{s} \quad$ Width of a spline or thickness of a module

c Vector containing unknowns $P_{c p}$ in the inversed Macaulay's method

cp Index for counting the number of control points

$\boldsymbol{d}, d_{j} \quad$ Distance of the $j^{\text {th }}$ gap between the $i$ and $i+1$ modules, where $i=1,2,3, \ldots, N_{m}$ and $j=1,2,3$, $\ldots, N_{m}-1$ for a tool with $N_{m}$ modules

$\boldsymbol{d}_{p}, d_{p, j p} \quad$ Distance of the $j p^{\text {th }}$ gap between the $c p$ and $c p+1$ control points, where $c p=1,2,3, \ldots, N_{p}$ and $j p=1,2,3, \ldots, N_{p}-1$ for a tool with $N_{p}$ control points

$h \quad$ Thickness of a sheet or a plate

$h_{\text {int }} \quad$ Thickness of an intermediate sheet

$h_{s} \quad$ Thickness of a spline

$i, j, k \quad$ Indexes for counting

jp Index for counting the number of gaps between control points

ks Index for counting the number of iterations in the spline shape optimisation process

$m s \quad$ Index for counting the number of data points in $R s, C s$ and $S$ 
$q \quad$ Uniformly distributed pressure load

$t_{1}, t_{2}, t_{3} \quad$ Time periods for the loading, creep-ageing and unloading steps in a creep-age forming process respectively

$u_{1}, u_{2}, u_{3} \quad$ Components of displacements in Cartesian coordinates

\section{$v \quad$ Poisson's ratio}

$\boldsymbol{w} \quad$ Vector of input displacements for the inversed Macaulay's method

$x_{1}, x_{2}, x_{3} \quad$ Cartesian coordinates

$x_{1, c p}, x_{2, c p}$, Cartesian coordinates of a specific control point, $x_{3, c p} \quad c p$

$C \boldsymbol{s}^{k s}, C s_{m s}^{k s} \quad$ Current spline shape at the $k s^{t h}$ iteration, where $m s=1,2,3, \ldots, N_{x 1}$ for a spline that has $N_{x 1}$ data points uniformly distributed along $S$

$E_{s} \quad$ Young's modulus of elasticity of a mechanical spline

$F_{s} \quad$ Flexural rigidity of a spline which is the product of multiplication of $E_{S}$ and $I_{S}$

$I_{s} \quad$ Second moment of area of a spline given by $I_{s}=$ $a_{s} h_{s}^{3} / 12$

K

$K_{1}, K_{2}$

$L_{\text {siter }}$

$M$

$N_{p}$

$N_{\text {siter }}$

$P_{c p}$
$\boldsymbol{R s}^{k s}, R s_{m s}^{k s}$

$\boldsymbol{S}, \boldsymbol{S}_{i}$

$T$

$\boldsymbol{X}_{c p}$

$\delta s^{k s}$

$\delta s_{\text {tol }}$

$\sigma_{y}$

$\sigma_{y, \text { int }}, \sigma_{y, s}$

$\sigma_{11}^{\max }$
Matrix of coefficients of equations for the inversed Macaulay's method

Integration constants

Limit of iterations that is set for the spline shape optimisation process

Bending moment

Total number of control points in a sparse-point tool

Number of iterations of the spline shape optimisation process

Reaction force at the $c p^{\text {th }}$ control point

Residual (vertical difference between the current spline's shape and the given shape to be

achieved) at the $k s^{\text {th }}$ iteration, where $m s=1,2,3$, $\ldots, N_{x 1}$ for a spline that has $N_{x 1}$ data points uniformly distributed along $\boldsymbol{S}$

Profile of a module containing a 2D point cloud in the $x_{1} x_{3}$ plane, where $i=1,2,3, \ldots, N_{m}$ for a tool with $N_{m}$ modules

Temperature at which creep-ageing or creep-age forming has taken place

Contains the position of each control point in the $x_{1} x_{3}$ plane

Minimised value of the largest absolute residual, $\min \left\{\max \left|\boldsymbol{R} \boldsymbol{s}^{k s}\right|\right\}$, at the $k s^{\text {th }}$ iteration of the spline shape optimisation process

Residual tolerance of the spline shape optimisation process

Yield strength of a material

Yield strengths of an intermediate sheet and a spline respectively

Maximum bending stress reached in a spline

\section{Introduction}

First developed in 1989 by Textron Aerostructures [1], creepage forming (CAF) is a sheet metal forming technique suitable for forming large integrally stiffened panels with complex curvature and abruptly changing thickness. CAF combines stress relaxation and age hardening into a single forming process, and the synchronous occurrence of the two phenomena reduces ongoing manufacturing cost significantly [2]. Benefitting from its unique process mechanisms, CA-manufactured parts require a low forming stress and contain low residual stresses which enhance fatigue resistance and improve strength-to-weight ratio of the final product [1].

At present, it is economically feasible to employ CAF to produce only dimensionally large parts in small batch quantities due to the long processing hours required. The latest research into CAF therefore is mainly focussed on the manufacture of aircraft wing panels. From the production of the Gulfstream IV's wing skins by Textron to the upper wing panels of the latest $£ 270 \mathrm{~m}$ Airbus (Toulouse, France) A380 superjumbo [3-6], CAF has already proven its practicality in the manufacturing of aircraft wing components. However, a number of challenges remain - one of which is the development of flexible CAF tools.

The need for flexible tools, particularly for manufacturing wing skin panels, is largely due to (i) the high cost of tools for forming large wing panels. For many years, aircraft manufacturers have been seeking for universal tooling that can form wing panels of different shape. Each aircraft wing has a unique aerofoil with complex requirements exclusive to a particular airplane model (Fig. 1), and specific tooling must be developed for each new wing design. Boeing (Chicago, USA) has revealed that the development of new tooling and facilities for each new airplane wing design commonly exceeds many millions of dollars [7]. Maintenance costs of the developed tool will also add-on over the production lifespan of an airplane. (ii) The need to compensate for springback of CAformed parts, which requires expensive and time-consuming shape rectification of conventional tools. Springback is a common problem amongst the production process of sheet metal parts. According to the figures provided by General Motors (Michigan, USA), the economic impact of delayed production and tooling revision costs due to springback alone was estimated to be over US\$50m a year in the automotive industry [8]. This is an even bigger problem when translated to CAF as springback of CA-formed parts can reach up to $80 \%$, and the abruptly changing thicknesses of wing panels also further complicates the mechanism of springback in practical operations [4, 9-11].

To enable springback to be predicted, a material and process model is necessary, and this must be based on comprehensive experimental data. These data must cover a wide range of test conditions including varying tool shape, part 


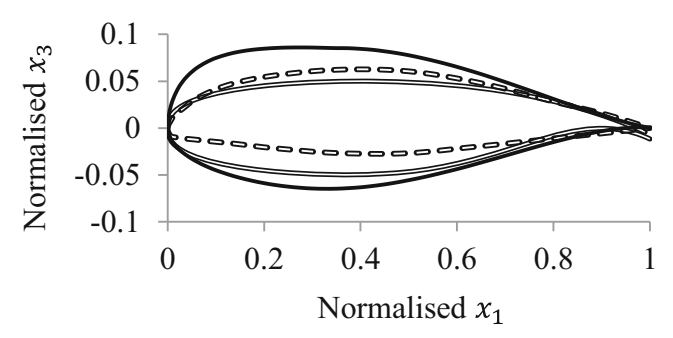

Fig. 1 Aerofoil profiles of three different aircraft

thickness and heat treatment cycle. Changing tool shape poses the biggest challenge of all and is responsible for the current lack of experimental data available for the validation of existing models. Modifying the tool shape of conventional solid-matched dies not only requires expensive precision apparatus and long hours of machining but also generates large amount of waste material as a result. Solid dies are also heavy in weight, which makes maintenance and handling of the tool difficult. Iteratively reworking the dies' surfaces to modify tool shape is therefore uneconomical and has led to the concept of flexible CAF tools.

Conventional flexible tools require the use of tightly packed forming pins to create a continuous forming surface; this is the theoretical base of a group of flexible dies that are characterised by arrays of discretised punch elements. One of the earliest organised activities with published results and validated conclusions on discrete dies were by Walczyk et al. in 1998 [12]. Based on their estimation for forming body panels of an F-14 fighter aircraft using a $1 \times 2 \mathrm{~m}$ forming tool, the cost of using a discrete die was approximated to be US $\$ 1.4 \mathrm{~m}$ with consumables inclusive, which was only $28 \%$ of the cost of using hard tooling [12]. The growing trend of discrete tooling with the lack of scientific development has led the authors to carry out further research on the design and analysis of discrete dies, where numerous examples had been given to illustrate the mechanics of discrete tooling [13]. This method of forming was coined the term 'multi-point forming (MPF)' by Li et al. in 1997, who later has led the MPF technique to find a wide range of applications, from shipbuilding to the manufacturing of titanium cranial prostheses [14-18].

Such conventional tools are heavy and unfavourable to CAF processes which require frequent transportation of the tooling. The number of pins in a flexible tool, or part count, is a key to the advancement of the current flexible tooling technology. Part count plays an important role as reducing it reduces both the weight and the costs of manufacturing and maintaining the tool at the same time. In this research, a flexible lightweight tool concept is introduced to control the tool shape using a series of spline shapes with fewer supporting points for each spline.

In this paper, an existing state-of-the-art flexible CAF tool is first described and analysed. The reader is then introduced to the concept of modularity in design and its application to the existing tooling. Applying the modular design concept to the existing tool partitions it into functional elements, and a proof-of-concept prototype is constructed using the functioning elements only, thus achieving part count reduction. Supported by a series of theoretical studies, subsequent evaluation of the prototype demonstrates the promising potential of this new method of design.

\section{A current state-of-the-art flexible CAF tool}

Literature on CAF-specific tool designs is scarce. An important contribution is the Airbus rib boards forming tool for forming aircraft wing panel components $[4,19]$. For the purpose of illustration, a schematic of the rib boards forming tool is shown in Fig. 2.

Figure 2a shows an isometric view of the tool, which makes use of two horizontal guide rails that are rigidly bolted on to a base (not illustrated). $N_{m}$ pairs of vertical guide rails are firmly supported by the horizontal guide rails and can slide only in the $x_{2}$ direction. Once slid to the required $x_{2}$, each pair of vertical guide rails is bolted to the horizontal guide rails, thus are structurally supported by both the horizontal guide rails and the base. The vertical guide rails from this instance onwards are firmly fixed in position. The $i^{\text {th }}$ and $(i+1)^{\text {th }}$ pairs of vertical guide rails are now spaced $d_{j}$ apart in the $x_{2}$ direction, where $i=1,2,3, \ldots, N_{m}$ and $j=1,2,3, \ldots,\left(N_{m}-1\right)$ for a tool with $N_{m}$ modules.

With reference to Fig. 2b, the $i^{\text {th }}$ module, which is a metal sheet with a profile described by $\boldsymbol{S}_{i}$, is chosen for each pair of vertical guide rails. Once positioned, each module is firmly bolted to the vertical guide rails. Each of $\boldsymbol{S}_{i}$ contains a 2D point cloud in the $x_{1} x_{3}$ plane and is chosen so that when all modules are positioned at the correct $x_{2}$, deflection surface of the uniformly loaded intermediate sheet, placed on top of all modules, it will form the shape of the required forming surface within predefined tolerances.

The positioned modules of thickness $a_{s}$, topped by the intermediate sheet and blank, are shown in Fig. 2c. This is a generic tool design such that modules with various profiles can be mounted to the vertical guide rails. The modules, vertical and horizontal guide rails and the base, which all together form a rigid supporting structure, are made of tooling steel that are substantially stiff and undergo negligible deformation during a CAF process.

In a typical CAF process, an intermediate sheet of $h_{\text {int }}$ thick is first placed on the rigid supporting structure. The intermediate sheet is made of spring-tempered steel with a yield strength, $\sigma_{y, \text { int }}$, sufficiently high to prevent it deforming plastically during CAF. A blank, which for the current aerofoil being produced, has a shape close to that of a trapezium, is then placed on the upper surface of the intermediate sheet. 
Fig. 2 Schematics of a 1Dmodularised CAF tool showing a an isometric view of the tool, $\mathbf{b}$ a single module and $\mathbf{c}$ view on cross-section A-A

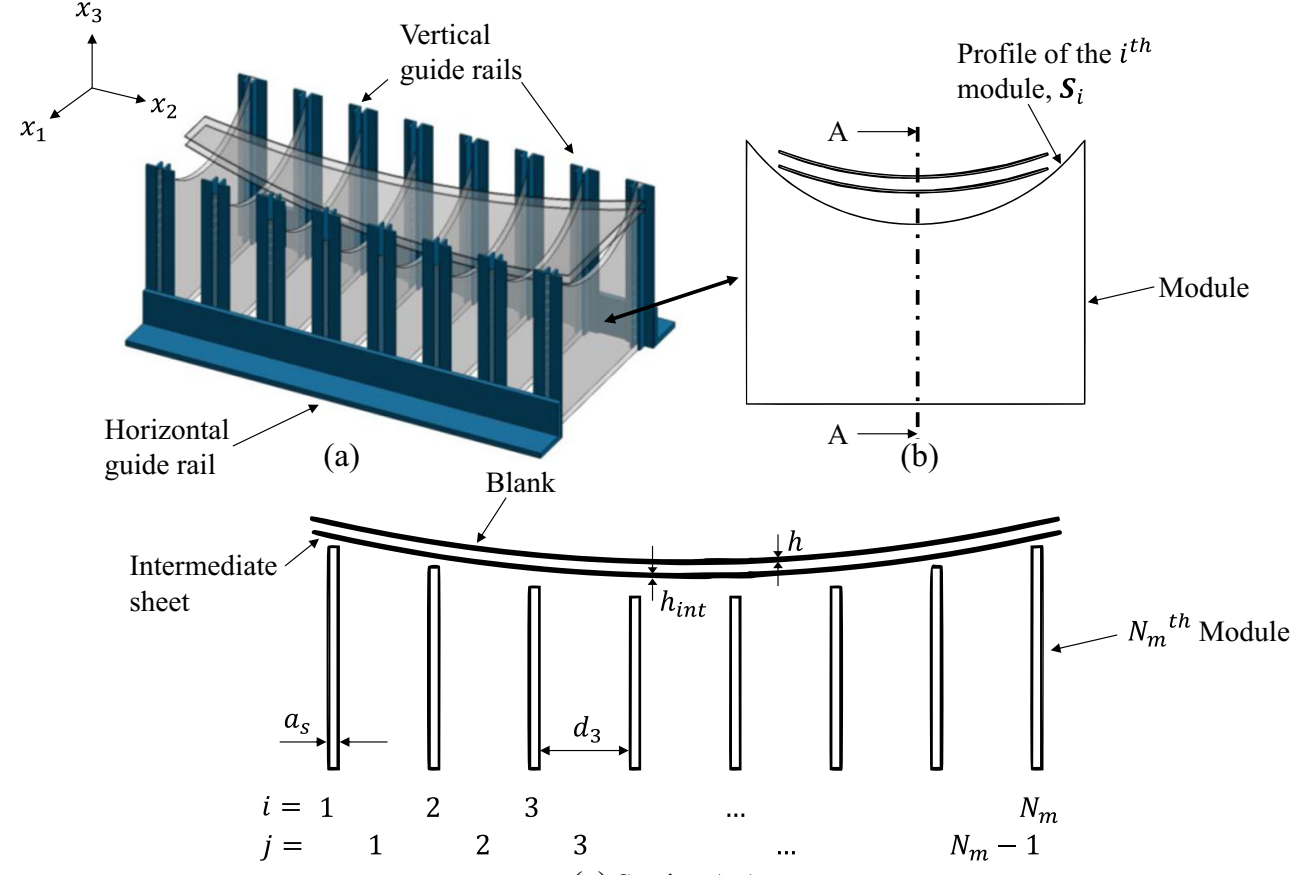

(c) Section A-A
With the use of vacuum bagging technique [1, 4, 20, 21], the uppermost surface of the blank is subjected to a uniformly distributed pressure load $q$ that acts towards negative $x_{3}$. Both the blank and the intermediate sheet deform towards negative $x_{3}$ as air is drawn away slowly over a duration of $t_{1}$. Final shapes of the blank and the intermediate sheet will conform to the shape defined by the modules, $\boldsymbol{S}_{i}$.

The deformed blank and intermediate sheet are then held at this configuration by $q$ whilst the whole apparatus is placed in an oven. The oven provides a heat treatment cycle to the whole apparatus at an elevated temperature, $T$, for a predetermined period of time, $t_{2}$. The blank undergoes plastic deformation during this period, as the material constituents of the blank precipitate, altering the microstructure of the alloy, thereby strengthening it $[1,5,22,23]$. $T$ and $t_{2}$ that characterise a heat treatment cycle are unique to each alloy and are set so that the CA-formed part will have final mechanical properties that meet the strength specification.

After time $t_{2}$, the oven is ventilated to allow the apparatus to return to room temperature. The cooled apparatus is then removed from the oven, and $q$ is slowly released over a period of $t_{3}$ as air returns back into the vacuumed environment. As a result of residual elastic stresses, the blank springs back to a shape somewhere between its original shape and the shape defined by $\boldsymbol{S}_{i}$. The intermediate sheet fully springs back to its original shape and is ready to be reused again. The pressure loading profile of a typical CAF process is illustrated in Fig. 3.

According to the inventor, A. Levers, the tool has led to a $50 \%$ reduction in tooling costs and more efficient processes for the production of wing panel components. These are the key factors that have made CAF practical for Airbus [4].

\section{Concept of modularity in design for flexible tooling}

Lever's tool has been designed by modularising the base structure of a conventional solid die in the $x_{2}$ direction [4], which results in the design that is only flexible in one dimension. From a design point of view, this is known as a 1Dmodularised tool design, and a number of published articles have already proven it practical for the CAF of large aircraft panel components [4, 24-27]. In this section, the flexible dimension of Lever's tool $\left(x_{2}\right)$ is retained, and flexibility of the tool is introduced to the other dimensions $\left(x_{1}\right.$ and $\left.x_{3}\right)$. The result is a 3D-modularised tool design concept with enhanced flexibility.

First consider a single module from the tool presented in Fig. 2. The subscripts $i$ and $j$ are dropped and the steps taken towards profile modularisation (Fig. 4) is described below.

Model idealisation Deletion/ modification of the geometric features are first carried out so that only the key geometric features of the original design are kept.

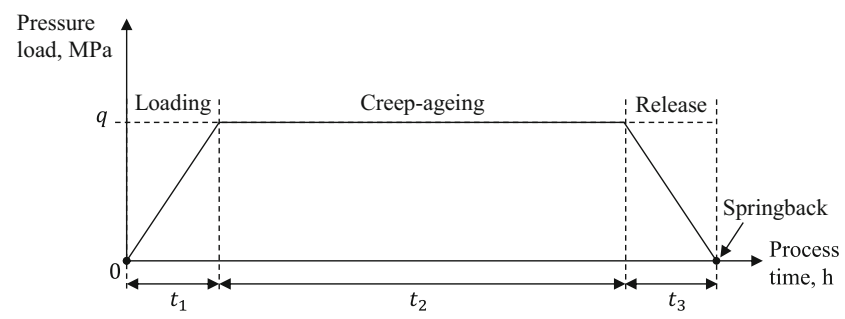

Fig. 3 Loading profile of a typical CAF process 
Fig. 4 Profile modularisation with mathematical splines and sparse controlling points

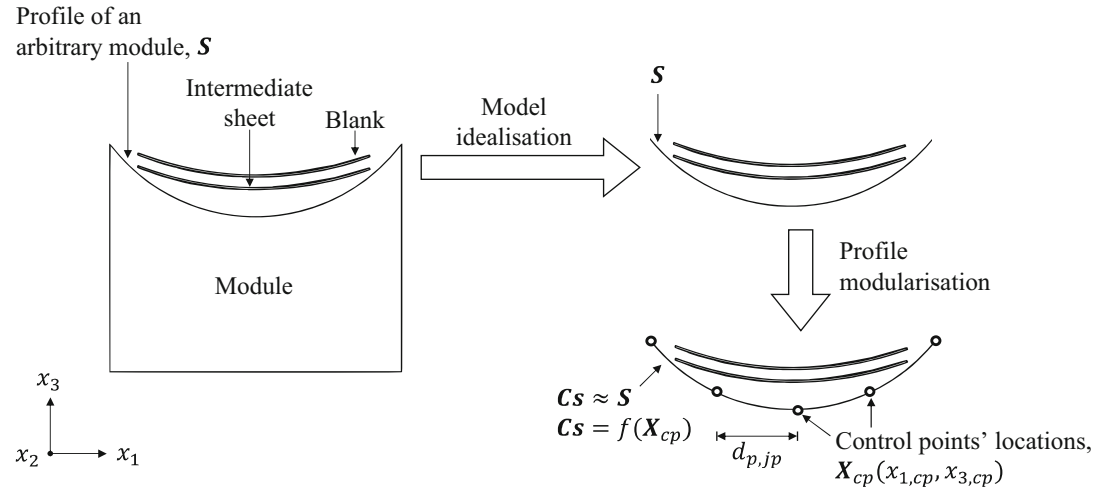

Profile modularisation Concept of modularity in design is applied to a single module profile. Profile $\boldsymbol{C s}$ is described by a mathematical spline and is a function of only the position of each control point, $\boldsymbol{X}_{c p}\left(x_{1, c p}, x_{3, c p}\right)$, in the $x_{1} x_{3}$ plane. $c p$ indicates the current control point that is being considered, such that $c p=1,2,3, \ldots, N_{p}$ for a spline that has $N_{p}$ control points. Distances between control points can be adjustable and the distance between the $c p^{\text {th }}$ and $(c p+1)^{\text {th }}$ controlling points is $d_{p, j p}$, where $j p=1,2,3, \ldots,\left(N_{p}-1\right)$.

During profile modularisation, a new parameter, $\boldsymbol{C s}$, is introduced which represents a shape that is a result of the positions of the controlling points in the $x_{1} x_{3}$ plane. From a theoretical standpoint, $\boldsymbol{C s}=\boldsymbol{S}$ (the target shape) can be achieved as $N_{p} \rightarrow \infty$ with $d_{p, j p} \rightarrow 0$, which in fact is the working principle behind conventional flexible tools. In reality however, an increase in $N_{p}$ must translate to an increase in part count, and this hinders conventional flexible tools from being used in CAF. Through a proof-of-concept prototype followed by a series of analyses, a method for designing flexible CAF tools using sparsely spaced controlling points (reducing $N_{p}$ whilst increasing $d_{p, j p}$ ) is evaluated in the remaining text.

\section{A proof-of-concept prototype and its characterisation}

In this section, a proof-of-concept prototype, manufactured for evaluating the proposed design, is presented. A corresponding analytical model is developed and experimentally validated. Finally, evaluation of the design method is conducted by comparing the validation results with that of the subsequent finite element (FE) simulations.

\subsection{Proof-of-concept prototype}

Figure 5 shows a generalised schematic of the testing prototype. The prototype has been manufactured with $N_{p}=5$ and $d_{p}$, $j p=120 \mathrm{~mm}$ for all $\mathrm{jp}$. A 600-mm-long spring-tempered steel strip (the mechanical spline) with dimensions of $a_{s}=35 \mathrm{~mm}$ and $h_{s}=1 \mathrm{~mm}$ in the $x_{2}$ and $x_{3}$ directions, respectively, is attached to and interpolates between all $c p$. Whilst $\boldsymbol{C s}$ of a mathematical spline (Fig. 4) depends only on $N_{p}$ and $\boldsymbol{X}_{c p}, \boldsymbol{C s}$ of a mechanical spline depends also on its flexural rigidity $\left(F_{s}\right)$ which is the product of multiplication between the spline's Young's modulus $\left(E_{s}\right)$ and second moment of area $\left(I_{s}=a_{s} h_{s}{ }^{3} /\right.$ 12). A novel spline-pins attachment method is conceived and employed, and once the spline is attached to the pins, the spline can slide only in the $x_{1}$ direction whilst $x_{3}$ is fixed by the sparse controlling points. As a result, $\boldsymbol{C s}$ changes as $x_{3, c p}$ are adjusted.

\subsection{Analytical modelling: inversed Macaulay’s method}

Based on the Euler-Bernoulli beam theory [28, 29], an analytical model (Fig. 6) is developed for the characterisation of the testing prototype. $\boldsymbol{X}_{c p}$ contains the position of each control pin
Fig. 5 A generalised schematic of the proof-of-concept prototype

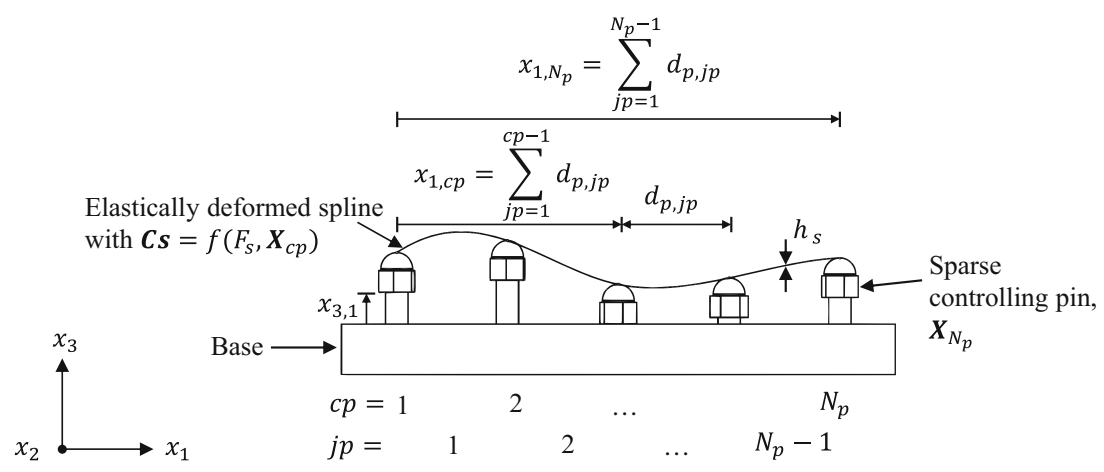




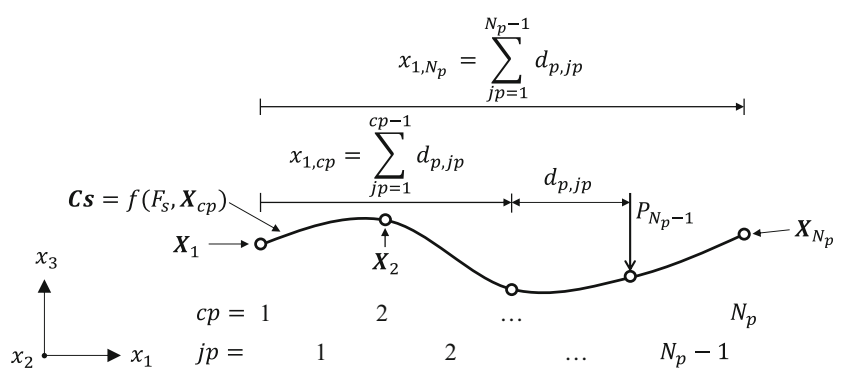

Fig. 6 A generalised Euler-Bernoulli beam model of the mechanical spline with sparse controlling points

in the $x_{1} x_{3}$ plane. $x_{1}$ of the leftmost control pin is set to, $x_{1,1}=0$ in all cases. Therefore, $x_{1, N_{p}}$ always represent the deformed spline's length. $\boldsymbol{C} \boldsymbol{s}$ can be displaced by altering $F_{s}$ and $\boldsymbol{X}_{c p}$.

Macaulay's method is adopted due to its generic form and is therefore easy to be implemented into computer programs [30]. For simplicity, its use is demonstrated here for the testing prototype which has $N_{p}=5$. However, it should be noted that this method is easily applicable to tools with any $N_{p}$. Now, for a spline with $N_{p}=5$, taking $M$ (moments) about the rightmost control point and applying Macaulay's function as described in Benham's work [29]

$M=P_{1} x_{1}+P_{2}\left\langle x_{1}-x_{1,2}\right\rangle+P_{3}\left\langle x_{1}-x_{1,3}\right\rangle+P_{4}\left\langle x_{1}-x_{1,4}\right\rangle$,

where $P_{c p}$ are the reaction forces at the $c p^{\text {th }}$ pin, where $c p=1,2, \ldots, 5$, and the Macaulay's functions (indicated by the angle brackets) can be integrated like ordinary functions. For example,

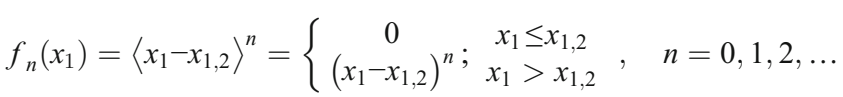

$\int_{0}^{x_{1}} f_{n}\left(x_{1}\right)=\frac{f_{n+1}\left(x_{1}\right)}{n+1}$.

Substituting Eq. 1 into

$F_{S} \frac{d^{2} x_{3}}{d x_{1}^{2}}=-M$

integrating twice and activating the Macaulay's function, $\boldsymbol{C s}$ can be obtained for each segment such that for $0 \leq x_{1} \leq x_{1,2}$

$x_{3}=\frac{1}{F_{s}}\left[-P_{1} \frac{x_{1}{ }^{3}}{6}+K_{1} x_{1}+K_{2}\right]$, for $x_{1,2} \leq x_{1} \leq x_{1,3}$

$x_{3}=\frac{1}{F_{s}}\left[-P_{1} \frac{x_{1}{ }^{3}}{6}-P_{2} \frac{\left(x_{1}-x_{1,2}\right)^{3}}{6}+K_{1} x_{1}+K_{2}\right]$,

for $x_{1,3} \leq x_{1} \leq x_{1,4}$

$x_{3}=\frac{1}{F_{s}}\left[-P_{1} \frac{x_{1}{ }^{3}}{6}-P_{2} \frac{\left(x_{1}-x_{1,2}\right)^{3}}{6}-P_{3} \frac{\left(x_{1}-x_{1,3}\right)^{3}}{6}+K_{1} x_{1}+K_{2}\right]$,

and for $x_{1,4} \leq x_{1} \leq x_{1,5}$

$\begin{aligned} x_{3}= & \frac{1}{F_{s}}\left[-P_{1} \frac{x_{1}^{3}}{6}-P_{2} \frac{\left(x_{1}-x_{1,2}\right)^{3}}{6}-P_{3} \frac{\left(x_{1}-x_{1,3}\right)^{3}}{6}-P_{4} \frac{\left(x_{1}-x_{1,4}\right)^{3}}{6}\right. \\ & \left.+K_{1} x_{1}+K_{2}\right],\end{aligned}$

where $K_{1}$ and $K_{2}$ are integration constants.

In practice, $x_{3, c p}$ are adjusted, and $\boldsymbol{C} \boldsymbol{s}$ is changed as a result. Classical beam equation, however, seeks forces as input and return $x_{3}, M$ and $\sigma_{11}$ along the length of the spline as outputs. A method must therefore be introduced to establish a set of displacement-based equations, so that the displacements at specific points on the beam (in this case, $x_{3, c p}$ ) become input parameters. $K_{1}, K_{2}$ and $P_{c p}$ together represent seven unknowns; seven equations are therefore needed for the equations to be solved. Together with the five displacement boundary conditions, the spline must also satisfy force and moment balance- these give the seven equations.

Solving for the unknowns individually can be a tedious task. It is more convenient to represent the seven equations in the form of a matrix

$$
\begin{gathered}
w(1,1) \\
\vdots \\
w(7,1)
\end{gathered}=\begin{array}{cccc}
K(1,1) & \cdots & K(1,7) & c(1,1) \\
\vdots & \ddots & \vdots & \vdots \\
K(7,1) & \cdots & K(7,7) & c(7,1)
\end{array} \text { or } \quad \boldsymbol{w}=\boldsymbol{K c}
$$

where $w(1,1) \ldots w(5,1)$ are the input displacements $x_{3, c p}$. $w(6,1)$ and $w(7,1)$ are set to zero in order to fulfil moment and force balances. $K(1,1) \ldots K(7,7)$ are the coefficients of equations. $c(1,1) \ldots c(5,1)$ are the unknown $P_{c p}$. $c(6,1)$ and $c(7,1)$ are the integration constants $K_{1}$ and $K_{2}$, respectively. The unknowns can now be obtained by inversing Eq. 9 so that 
Table 1 Individual height of the pins in the three test cases

\begin{tabular}{llrllllr}
\hline \multirow{2}{*}{ Shape description } & \multicolumn{2}{c}{$x_{3, c p}, \mathrm{~mm}$} & & & \\
\cline { 3 - 7 } & & $c p=1$ & 2 & \multicolumn{2}{l}{3} & \multicolumn{1}{l}{4} & \multicolumn{1}{c}{5} \\
\hline Test case 1 & Symmetric U-shape & 32.50 & 10.00 & 0.00 & 10.00 & 32.50 \\
Test case 2 & Symmetric W-shape & 8.00 & 0.00 & 3.00 & 0.00 & 8.00 \\
Test case 3 & Anti-symmetric & 15.00 & 10.00 & 3.00 & 6.00 & 0.00 \\
\hline
\end{tabular}

$\boldsymbol{c}=\boldsymbol{K}^{-1} \boldsymbol{w}$

This is the inversed Macaulay's method (IMM), and as $c$ is solved, a solution is reached for any given set of $x_{3, c p}$. $\boldsymbol{C s}$ can now be obtained by calculating $x_{3}$ everywhere along the spline. Using MATLAB (vR2012b, MathWorks, Natick, MA, USA) for matrix handling, $\boldsymbol{C s}$ due to $x_{3, c p}$ for the three test cases listed in Table 1 is computed. The chosen test cases aim to simulate different scenario, and stress analyses were conducted to ensure each bending curvature is valid - the solution is valid as long as the maximum bending stress in the spline remains smaller than its yield strength, $\sigma_{11}^{\max }<\sigma_{y, s}$. Values of $N_{p}, d_{p}$ and $I_{s}$ identical to that of the prototype were used and $E_{s}=207 \mathrm{GPa}$ in all cases.

\subsection{Experimental validation}

Results due to the analytical solution have been experimentally validated using the prototype. Initially, $x_{3, c p}$ was set to zero for all $c p$; the spline was then attached to the pins. $x_{3}$ was slowly adjusted for each $c p$ and continuously monitored by a digital height gauge with an accuracy of $\pm 0.01 \mathrm{~mm}$ throughout the adjustment process.

With $x_{3, c p}$ correctly adjusted for all $c p$, the resulting $\boldsymbol{C s}$ between $x_{1,1}$ and $x_{1,5}$ was measured using a coordinate

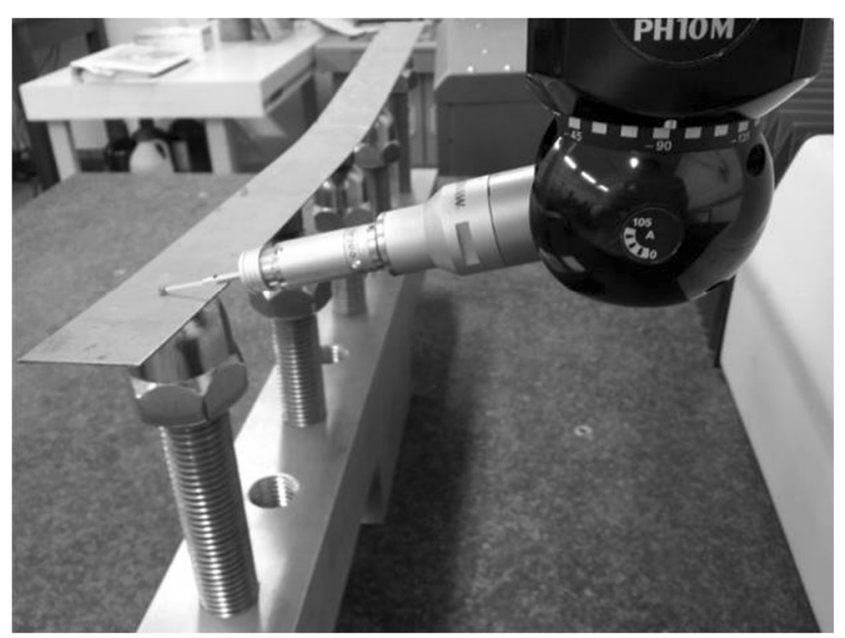

Fig. 7 Spline shape measurement using a coordinate measuring machine measuring machine (CMM LK G90C, Nikon Metrology, Derby, England, UK) as shown in Fig. 7. Three sets of measurements per test case have been recorded, and the mean shape was used.

\subsection{FE simulation}

Corresponding FE analyses were carried out using PAMSTAMP (v2G 2012.0, ESI Group, Paris, France). Geometric models of the forming pins were generated using SolidWorks (v2012 Education Edition, Dassault Systèmes SolidWorks, Waltham, MA, USA) as IGES files v5.3 and imported into PAM-STAMP as rigid bodies through the "Tools" module. The spline was represented by a $600 \times 35 \mathrm{~mm}$ geometric model created in the "Blank" module and meshed using Belytschko-Tsay shell elements in second strain order. Gauss rule was used with 10 integration points through the 1-mmthick shell elements. Mechanical properties of the spline identical to those used for analytical modelling were assigned with a Poisson's ratio of $v=0.3$. Cartesian kinematics of the pins was locked in all directions except in $x_{3}$, which were userdefined displacements to match those in Table 1. Frictionless contacts between the spline and pins were defined. FE simulated results were then evaluated with the analytical and experimental results.

\subsection{Evaluation}

In a conventional wing skin panel shape inspection process, a formed panel is lowered by an overhead crane and placed on a mechanical fixture - a tool that is used to ensure the formed panel is within the required assembly tolerance. Although different requirements may be given, a vertical distance representing the size of the gap between the formed component and the contour of the check fixture is usually considered an acceptable requirement for large aircraft components [31]. For example, an assembly tolerance of $-0,+1 \mathrm{~mm}$ is required for the Airbus A380 wing skin [4], and the panel must be bent to within this tolerance to ensure the panel will not be overstressed during the subsequent assembling procedure. Based on the conventional use of this original definition by aircraft manufacturers and its easy-to-interpret nature, vertical difference between experimental and modelling results is used for profile evaluation.

Comparisons of the simulated profiles (FE) with the analytical (IMM) and experimental (Exp.) profiles are shown in Fig. 8. Good correlation between the deflection profile $\left(u_{3}\right)$ of the three can be seen. Vertical differences between $u_{3}$ of the experimental and the IMM or FE results are all found to be bounded by $\pm 1 \mathrm{~mm}$. This shows that the method is suitable for designing flexible CAF tools with less than $\pm 1 \mathrm{~mm}$ error in the forming surface. 
Fig. 8 A comparison of the experimental data with analytically (IMM) and FEpredicted spline shapes for the three test cases
Test case 1

Test case 2
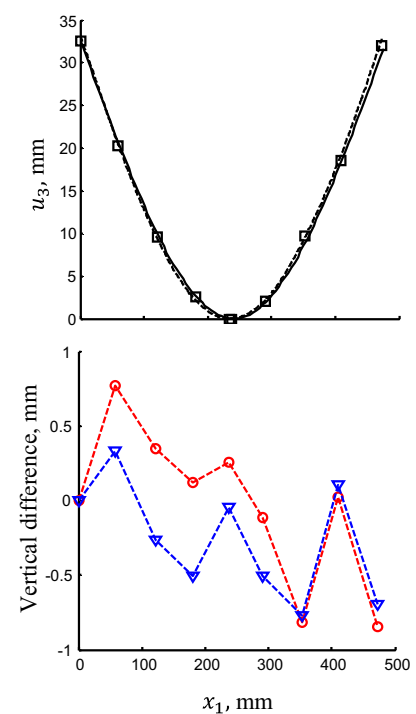

Test case 3

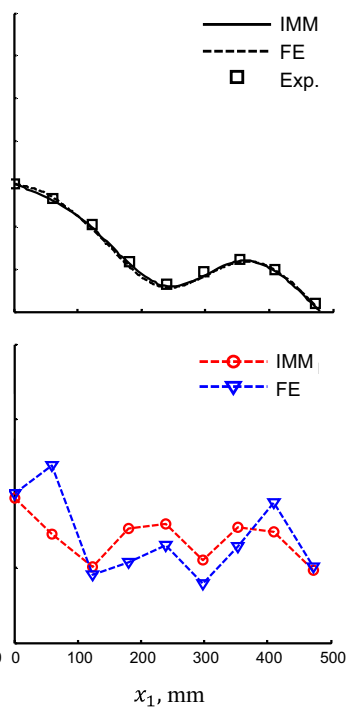

\section{Solution to a pseudo-rigid spline with shape $C s \approx S$ under pressure $q$}

Successful implementation of this design method must require $\boldsymbol{C s} \approx \boldsymbol{S}$ within an acceptable shape tolerance $\left(\delta s_{t o l}\right)$ under any $q$ that is experienced by the spline in a typical CAF process (Fig. 3). Introducing an additional term $\left(q x_{1,5}{ }^{2} / 2\right)$ to Eq. 1

$$
\begin{aligned}
M= & P_{1} x_{1}+P_{2}\left\langle x_{1}-x_{1,2}\right\rangle+P_{3}\left\langle x_{1}-x_{1,3}\right\rangle+P_{4}\left\langle x_{1}-x_{1,4}\right\rangle \\
& +\left(q x_{1,5}^{2} / 2\right),
\end{aligned}
$$

where $\left(q x_{1,5}{ }^{2} / 2\right)$ represents the contribution of load $q$, uniformly distributed over the total span of the spline, $x_{1,5}$, to the moment. Equations of similar form to that of Eqs. 5 to 8 (and thus $\boldsymbol{C s}$ ) can be obtained by following the same treatment as above together with the consideration of $q$ in the moment and force balances. The result is an analytical model for a spline with $\boldsymbol{C s}$ controlled by $F_{s}$ and $\boldsymbol{X}_{c p}$, that is uniformly loaded by $q$.

It is a requirement to simulate a rigid spline whose shape can remain $\boldsymbol{C s} \approx \boldsymbol{S}$ under the action of $q$. The idea of rigidity is such that under any applied $q$, stiffness of the spline is so high that either its shape does not change, or the change is negligible. Thus, it can be hypothesised that by carrying out optimisation on certain process parameters, a solution to a pseudo-rigid spline with shape $\boldsymbol{C} \approx \boldsymbol{S}$ can be reached within the required $\delta s_{t o l}$. Based on this hypothesis, three parameters that can cause $\boldsymbol{C s}$ to deviate from given $\boldsymbol{S}$ are identified and optimised-these are $N_{p}$, $x_{1, c p}$ and $h_{s}$.
Trust-region optimisation (TRO) algorithm is employed to optimise $\boldsymbol{C} \boldsymbol{s}^{k s}$, where $k s=1,2,3, \ldots, N_{\text {siter }}$ for an optimisation process with $N_{\text {siter }}$ iterations. The fourstep spline shape optimisation process illustrated in Fig. 9 is described here.

Step 1 Definition: Define $a_{s}, x_{1, N_{p}}, E_{s}, q, \sigma_{y, s}, \boldsymbol{S}$ for a specific case. Limit of iterations, $L_{\text {siter }}$, and $\delta s_{\text {tol }}$ also need to be specified.

Step 2 Initiation: Assignment of initial values $N_{p}^{0}$ and $h_{s}^{0}$. $x_{1, c p}^{0}$ is then automatically initiated by evenly distributing $N_{p}^{0}$ control points along $\boldsymbol{S}$.

Step 3 IMM: Compute the initial spline shape $\left(\boldsymbol{C s}{ }^{0}\right)$ based on the given definition and initial values.

Step 4 TRO: Compute the residual $\left(\boldsymbol{R} \boldsymbol{s}^{k s}\right)$ at the $k s^{t h}$ iteration of an optimisation process, which represents the vertical difference between the current spline's shape $\left(\boldsymbol{C} \boldsymbol{s}^{k s}\right)$ and the given shape to be achieved $(\boldsymbol{S})$. This is given by

$$
\begin{aligned}
\boldsymbol{R s}^{k s} & =R s_{m s}^{k s}=\boldsymbol{C} \boldsymbol{s}^{k s}-\boldsymbol{S}, \\
k s & =1,2,3, \ldots, N_{\text {siter }}
\end{aligned}
$$

where $m s=1,2,3, \ldots, N_{x 1}$ for a spline that has $N_{x 1}$ data points uniformly distributed along $\boldsymbol{S}$ (Fig. 9). Least squares and infinity norm [32] given by

$\min \left\{\left\|\boldsymbol{R} \boldsymbol{s}^{k s}\right\|^{2}\right\}=\min \left\{\sum_{m s=1}^{N_{x 1}}\left(R s_{m s}^{k s}\right)^{2}\right\}$ 


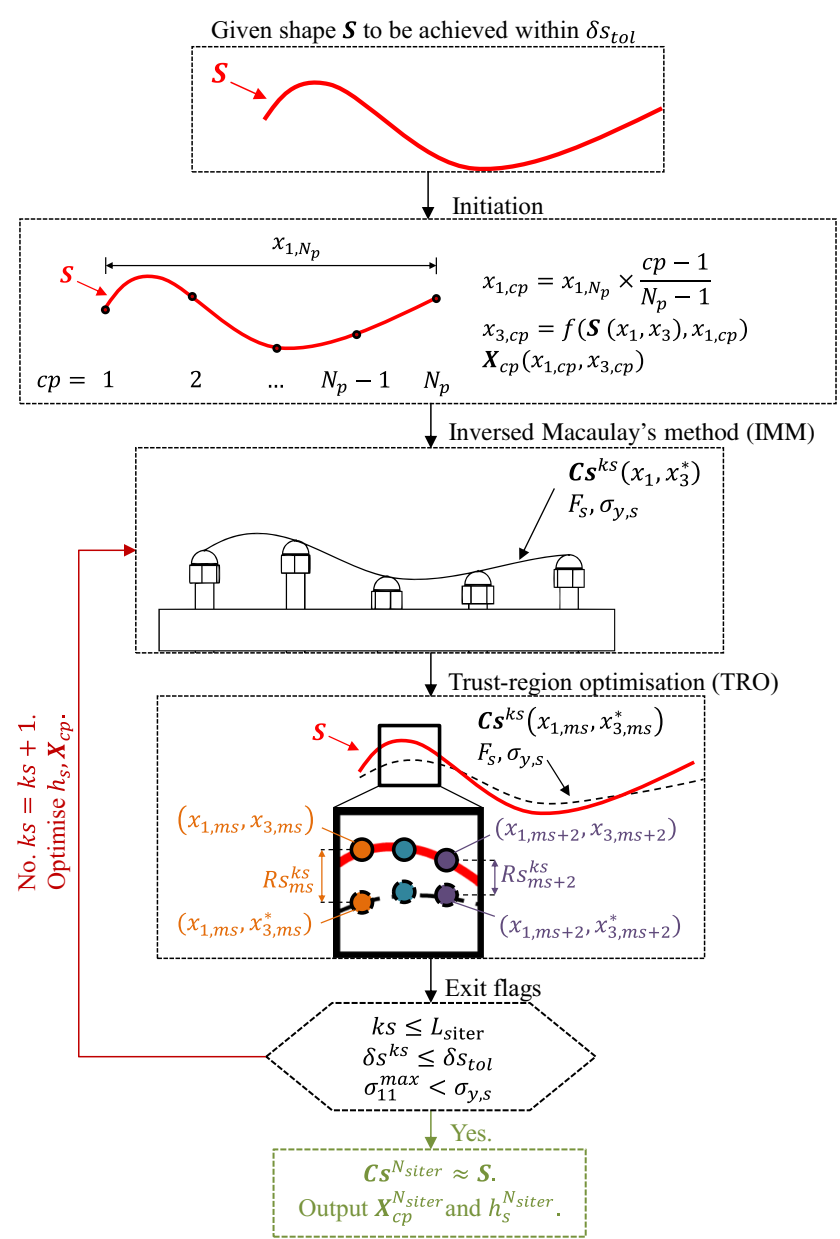

Fig. 9 Flow diagram of the integrated optimisation process for tool offsetting

and

$\delta s^{k s}=\min \left\{\left\|\boldsymbol{R} s^{k s}\right\|_{\infty}\right\}=\min \left\{\max _{m s}\left|R s_{m s}^{k s}\right|\right\}$,

respectively, are used concurrently for monitoring the minimisation of $\boldsymbol{R} \boldsymbol{s}^{k s}$. As $\delta s^{k s<L_{\text {sitit }}} \leq \delta s_{t o l}$ is reached with $\sigma_{11}^{\max }<\sigma_{y, s}$, a valid solution is arrived to a pseudo-rigid spline with dimensions $h_{s}$ (thickness), $a_{s}$ (width) and $x_{1, N_{p}}$ (length) that is uniformly loaded by $q$ and has shape $\boldsymbol{C s} \approx \boldsymbol{S}$.
Table 2 Assigned parameters in steps 1 and 2 of the integrated optimisation process

\begin{tabular}{lllllllll}
\hline $\begin{array}{l}a_{s}, \\
\mathrm{~mm}\end{array}$ & $\begin{array}{l}x_{1, N_{p}}, \\
\mathrm{~mm}\end{array}$ & $\begin{array}{l}E_{s}, \\
\mathrm{MPa}\end{array}$ & $\begin{array}{l}q, \\
\mathrm{~N} \mathrm{~mm}^{-1}\end{array}$ & $\begin{array}{l}\sigma_{y, s}, \\
\mathrm{MPa}\end{array}$ & $\begin{array}{l}L_{s i t e r} \\
\delta s_{\text {tol }}, \\
\mathrm{mm}\end{array}$ & $\begin{array}{l}N_{p}^{0} \\
h_{s}^{0}, \\
\mathrm{~mm}\end{array}$ \\
\hline 35 & 500 & 207000 & 1 & 1014 & 500 & 1 & 3 & 1 \\
\hline
\end{tabular}

\section{Case studies}

Implementing the above procedures in MATLAB, parameters in Table 2 are assigned. Two cases are studied whose $\boldsymbol{S}$ are described by

Case $\boldsymbol{S}_{1}: x_{3}=\frac{\left(x_{1}-250\right)^{2}}{3 \times 10^{3}}$,

Case $\quad \boldsymbol{S}_{2}: x_{3}=70 \sin \left(\frac{x_{1}}{50}\right) e^{-\frac{x_{1}}{300}}$

\section{Results}

Results from the case studies are presented in Figs. 10 and 11. Figure 10 compares deflections and normalised residuals of $\boldsymbol{S}$ with $\boldsymbol{C s}$ before and after tool offsetting for case $\boldsymbol{S}_{1}$. Results for case $\boldsymbol{S}_{2}$ are shown in Fig. 11. Key outputs from the optimisation code are tabulated in Table 3.

\section{Discussion}

$S_{1}$ simulates a general curvature that is similar to those seen in the aerofoils of a typical aircraft (Fig. 1). $\delta s$ has been optimised from 22.71 to $0.37 \mathrm{~mm}$ and $h_{s}$ has changed from 1 to $2.58 \mathrm{~mm}$ after nine iterations. Three control points are sufficient to achieve $\boldsymbol{S}_{1}$, and $\boldsymbol{X}_{c p}$ has only moved slightly from their original positions. $\sigma_{11}^{\max }=209.5 \mathrm{MPa}$, which means that the deformation is elastic and the solution is therefore valid.
Fig. 10 Case $\boldsymbol{S}_{1}$ : comparing a $u_{3}$ and $\mathbf{b} \boldsymbol{R} \boldsymbol{s} / \delta s_{t o l}$ of $\boldsymbol{S}_{1}$ with $\boldsymbol{C s}^{0}$ and $\mathrm{Cs}^{9}$ (a)

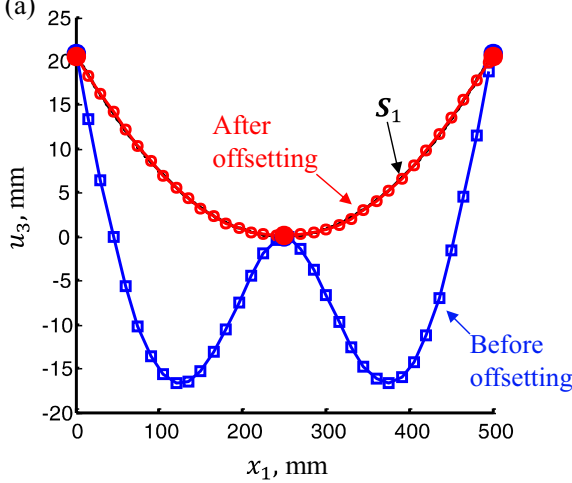

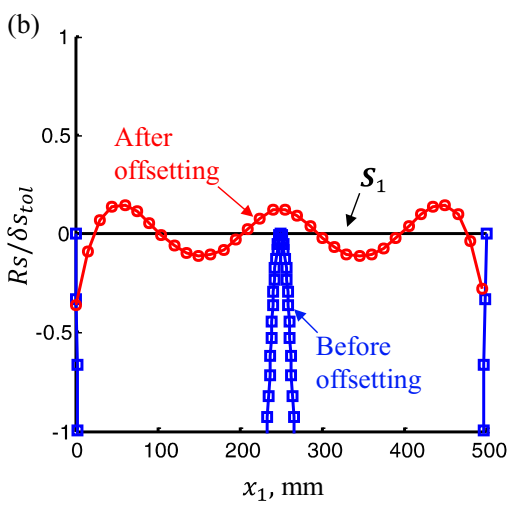


Fig. 11 Case $\boldsymbol{S}_{2}$ : comparing $u_{3}$ and $\boldsymbol{R s} / \delta s_{t o l}$ of $\boldsymbol{S}_{2}$ with $\boldsymbol{C} \boldsymbol{s}^{0}$ and $\boldsymbol{C s}^{N_{\text {sititr }}}$. a and $\mathbf{b}$ show that the solution cannot converge within $L_{\text {siter }}=500$ with only three controlling points. $\mathbf{c}$ and $\mathbf{d}$ show $\delta s_{t o l}$ is achieved after introducing two extra control points
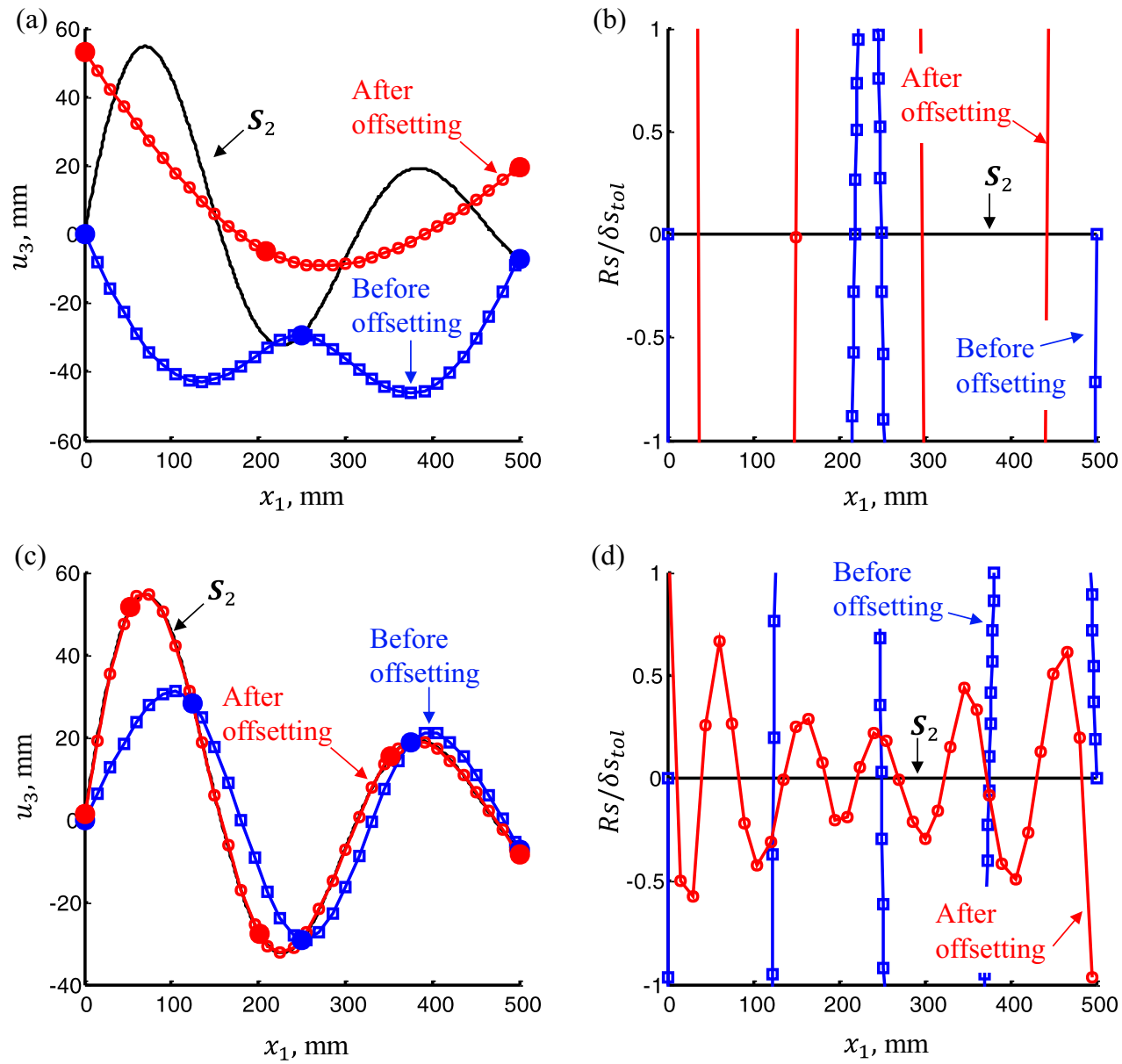

(d)

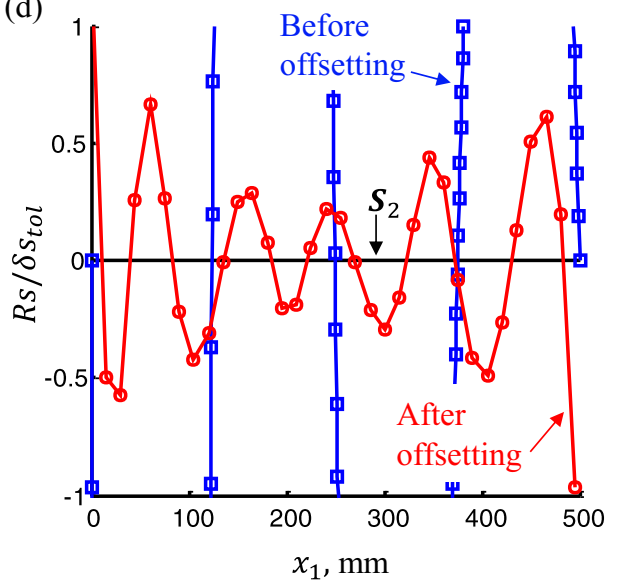

$\boldsymbol{S}_{2}$ aims to achieve a more complex shape with continuously changing curvatures. From Fig. 11a, one can immediately see that three control points are insufficient for $\boldsymbol{S}_{2}$. Despite $\boldsymbol{X}_{c p}$ have been displaced significantly and $h_{s}$ has increased from 1 to $5 \mathrm{~mm}, \delta s$ has changed only from 89.32 to $52.93 \mathrm{~mm}$ upon reaching $N_{\text {siter }}-\boldsymbol{C s} \approx \boldsymbol{S}$ could not be achieved as a result. Introduction of two additional controlling points are found to be necessary in order for the required $\boldsymbol{S}_{2}$ to be achieved. Figure 11c shows $\boldsymbol{S}_{2}$ is achieved by optimising $h_{s}$ and $\boldsymbol{X}_{c p}$ with $N_{p}=5$. Final $\delta s$ is $0.97 \mathrm{~mm}$, and $h_{s}$ has changed from 1 to $1.14 \mathrm{~mm}$. However, this solution was found invalid because $\sigma_{11}^{\max }=1903.4 \mathrm{MPa}$, which is greater than $\sigma_{y, s}$.

Case $\boldsymbol{S}_{1}$ shows a classic example of how the current design method can be used for forming structures that have a general curvature, such as that seen in the aerofoils of typical aircraft wing skins. From this study, it can be seen that the deviation of $\boldsymbol{C S}$ from $\boldsymbol{S}$ can be controlled by changing only $h_{s}$.

Because the functioning of the current design and the corresponding theoretical studies rely heavily on the EulerBernoulli beam theory, the assumptions that the theory is based on also pose several limitations on the current design method. This can be overcome by extending or replacing the Euler-Bernoulli beam theory to account for what is needed.
For example, one may use the Timoshenko beam theory to account for shear effects. Stephen's work in 2007 already demonstrated how it can be coupled with the Macaulay's method [33], but discussion of this is beyond the scope of this paper.

As mentioned earlier, $\boldsymbol{C} \boldsymbol{s}=\boldsymbol{S}$ can be achieved as $N_{p} \rightarrow \infty$, and this is the theoretical base of conventional discrete tooling. The key advantage of this latest method of tool design over conventional discrete tooling is its lightweight design. Taking the prototype manufactured for this study as an example, the $600 \times 60 \times 60 \mathrm{~mm}$ steel base weighs $17 \mathrm{~kg}$. It was mounted with five controlling pins, each weighs $1 \mathrm{~kg}$. Together with the $600 \times 35 \times 1 \mathrm{~mm}$ spline, the testing prototype has a total weight of just over $22 \mathrm{~kg}$. For a conventional discrete

Table 3 Key outputs from the optimisation code

$$
N_{p} \quad N_{\text {siter }} \quad \delta s^{0}, \mathrm{~mm} \quad \delta s^{N_{\text {siter }}, \mathrm{mm}} \quad h_{s}^{N_{\text {siter }}}, \mathrm{mm} \quad \sigma_{11}^{\max }, \mathrm{MPa}
$$

\begin{tabular}{llllrll}
\hline Case $\boldsymbol{S}_{1}$ & 3 & 9 & 22.71 & 0.37 & 2.58 & 209.50 \\
Case $\boldsymbol{S}_{2}$ & 3 & 500 & 89.32 & 52.93 & 5.00 & - \\
Case $\boldsymbol{S}_{2}$ & 5 & 13 & 30.02 & 0.97 & 1.14 & 1903.4 \\
\hline
\end{tabular}


tool, the same base can accommodate 12 more pins of the same size, and assuming each pin has a volume of $142 \mathrm{cc}$, this represents an increase of approximately $55 \%$ in tooling weight and $59 \%$ in volume of material used comparing with the current prototype. Not only is the present design cheaper but it is also easier to maintain due to less material used and its lightweight design. Fewer pins also means shorter set-up time is required; this will result in a significant reduction in the manufacturer's ongoing costs [7, 34], justifying the tool's return on investment. Considering the typical application of CAF is for manufacturing aircraft wing panels and a typical wing skin panel is about $33 \mathrm{~m}$ long with a maximum width of $2.7 \mathrm{~m}$ [19], the amount of weight reduced at such a large scale will be far more substantial. This can largely influence the decision made by aircraft manufacturers as to whether or not to adopt CAF as a new forming process [7].

Although a flexible tool, the major shortcoming of the rib boards design illustrated in Fig. 2 is also its flexibility. Whilst the tool is flexible in $x_{2}$, aerofoil of the wing panel that is to be formed remains determined by the shape, $\boldsymbol{S}$, that is machined into each module. This means for every new wing design, or any adjustments that are required for springback compensation, a new set of modules must be made. The economic impact of such practice is already highlighted earlier in this article - with two extra degrees of freedom in the $x_{1}$ and $x_{3}$ directions, the present tool design will not have such problem.

\section{Conclusions}

A method for designing flexible $\mathrm{CAF}$ tools was conceived and evaluated using a prototype that has been manufactured for this study. Theoretical studies were carried out in order to characterise the prototype. An integrated optimisation process for tool offsetting was then introduced, and its use was also demonstrated. It was confirmed that CAF tools with less than $\pm 1 \mathrm{~mm}$ error in the forming surface can be achieved with the proposed design method. Furthermore, this error can in theory be compensated, and thus eliminated from the final CA-formed parts, using the proposed tool offsetting method. This novel design concept can now be used to construct lightweight and flexible CAF tools.

\begin{abstract}
Acknowledgements The strong support from Aviation Industry Corporation of China (AVIC) Beijing Aeronautical Manufacturing Technology Research Institute (BAMTRI) for this funded research is much appreciated. The research was performed at the AVIC Centre for Structural Design and Manufacture at Imperial College London.
\end{abstract}

Conflict of interest The authors declare that there is no conflict of interest.

Open Access This article is distributed under the terms of the Creative Commons Attribution License which permits any use, distribution, and reproduction in any medium, provided the original author(s) and the source are credited.

\section{References}

1. Holman MC (1989) Autoclave age forming large aluminum aircraft panels. J Mech Work Technol 20:477-488

2. Zhan L, Lin J, Dean TA (2011) A review of the development of creep age forming: experimentation, modelling and applications. Int $\mathrm{J}$ Mach Tools Manuf 51(1):1-17

3. Airbus Press Office (2013) Airbus Aircraft 2013 Average List Prices (mio USD). http://www.airbus.com/presscentre/corporateinformation/key-documents/?docID=14849\&eID=dam_frontend push. Accessed 24 March 2014

4. Levers A (2003) Jumbo processes. IEE Manuf Eng 82(3):42-45

5. Jeunechamps P-P, Ho KC, Lin J, Ponthot J-P, Dean TA (2006) A closed form technique to predict springback in creep age-forming. Int J Mech Sci 48(6):621-629

6. Eberl F, Gardiner S, Campanile G, Surdon G, Venmans M, Prangnell P (2008) Ageformable panels for commercial aircraft. Proc Inst Mech Eng Part G J Aerosp Eng 222(6):873-886

7. Woods QT (1990) Assembly jig and method for making wing panels. PAT US 4,894,903. 23 Jan 1990

8. Gan W, Wagoner RH (2004) Die design method for sheet springback. Int J Mech Sci 46(7):1097-1113

9. Pitcher PD, Styles CM (2000) Creep age forming of 2024A, 8090 and 7449 alloys. Mater Sci Forum 331-337:455-460

10. Ho KC, Lin J, Dean TA (2004) Modelling of springback in creep forming thick aluminum sheets. Int J Plast 20(4-5):733-751

11. Yang H, Davies CM, Lin J, Dear JP (2013) Prediction and assessment of springback in typical creep age forming tools. Proc Inst Mech Eng Part B J Eng Manuf 227(9):1340-1348

12. Walczyk DF, Lakshmikanthan J, Kirk DR (1998) Development of a reconfigurable tool for forming aircraft body panels. J Manuf Syst 17(4):287-296

13. Walczyk DF, Hardt DE (1998) Design and analysis of reconfigurable discrete dies for sheet metal forming. J Manuf Syst 17(6):436-454

14. Li M, Liu Y, Su S, Li G (1999) Multi-point forming: a flexible manufacturing method for a 3-D surface sheet. J Mater Process Technol 87(1-3):277-280

15. Liu C, Li M, Fu W (2006) Principles and apparatus of multi-point forming for sheet metal. Int J Adv Manuf Technol 35(11-12):12271233

16. Tan FX, Li MZ, Cai ZY (2007) Research on the process of multipoint forming for the customized titanium alloy cranial prosthesis. $\mathrm{J}$ Mater Process Technol 187-188:453-457

17. Chen J-J, Li M-Z, Liu W, Wang C-T (2004) Sectional multipoint forming technology for large-size sheet metal. Int J Adv Manuf Technol 25(9-10):935-939

18. Chen J-J, Liu W, Li M-Z, Wang C-T (2005) Digital manufacture of titanium prosthesis for cranioplasty. Int J Adv Manuf Technol 27(1112):1148-1152

19. Levers A (2008) Aircraft component manufacturing tool and method. PAT EP 1581357 B1. 26 Mar 2008

20. Levers A, Wiles G (2011) Assembling and shaping laminate panel. PAT US 2011/0143100 A1. 16 Jun 2011

21. Inforzato DJ, Costa PR Jr, Fernandez FF (2012) Creep-age forming of AA7475 aluminum panels for aircraft lower wing skin application. Mater Res 15(4):596-602

22. Lin J, Ho KC, Dean TA (2006) An integrated process for modelling of precipitation hardening and springback in creep age-forming. Int J Mach Tools Manuf 46(11):1266-1270

23. Zhu A, Starke EA Jr (2001) Materials aspects of age-forming of AlxCu alloys. J Mater Process Technol 117:354-358

24. Newkirk TL, Holman MC (1998) Method and apparatus for constructing a complex tool surface for use in an age forming process. PAT US 5,729,462. 17 Mar 1998 
25. Brewer HM Jr, Holman MC (1992) Method of tool development. PAT US 5,168,169. 1 Dec 1992

26. Bornschlegl H, Kohler W (2001) Process for forming a plate-like component. PAT US 6,264,771. 24 Jul 2001

27. Airbus Group (2014) Creep forming. Airbus Technology Licensing. http://www.technology-licensing.com/etl/int/en/What-we-offer/ Technology-videos.html. Accessed 21 March 2014

28. Timoshenko SP (1953) History of strength of materials. McGrawHill Book Company, Inc., New York

29. Benham PP, Crawford RJ, Armstrong CG (1996) Mechanics of engineering materials, 2nd edn. Education, Pearson
30. Macaulay WH (1919) Note on the deflection of beams. Messenger Math 48:129-130

31. Phillips AL, Johnson WT, Crawford GL, Barnett TD, Scriber LJ, Breidenbach DI (2006) Stringer check fixture and method. PAT US 7103985 B1. 12 Sep 2006

32. Moler CB (2004) Numerical computing with MATLAB. Society for Industrial and Applied Mathematics

33. Stephen NG (2007) Macaulay's method for a Timoshenko beam. Int J Mech Eng Educ 35(4):285-292

34. Swift KG, Booker JD (2003) Process selection from design to manufacture. $2^{\text {nd }}$ Ed. Butterworth-Heinemann 